\section{DIE INTERNATIONALEN ERBEN DES BAUHAUSES IM FOKUS / BAUHAUS PROJECT: FOCUS ON INTERNATIONAL STYLE HEIRS}

\author{
Kaija Voss, \\ https://orcid.org/0000-0003-3126-8029 \\ PhD in History of architecture, \\ Scientific assistant, \\ Brandenburgische Technische \\ Universität Cottbus, \\ Technische Universität Dresden, \\ Lecturer for Deutsche Stiftung \\ Denkmalschutz, \\ Germany \\ architektur-sehenlernen@gmx.de \\ Jean Molitor, \\ jean.molitor.photography \\ Artist-photographer, \\ Author of the Project Bau1haus, \\ Berlin, Germany \\ jean.molitor@bau1haus.de
}

ПPOEKT BAU1HAUS: ФОКУС

\section{НА МІЖНАРОДНИХ СПАДКОЄМЦЯХ} стилю

\section{Кайя Восс,}

https://orcid.org/0000-0003-3126-8029

доктор фрілософрії з історії архітектури, науковий співробітник

Брандебурзького технічного університету в Котбусі,

Дрезденського технічного університету, доцент Німецької фундації охорони пам'яток, Німеччина

architektur-sehenlernen@gmx.de

\section{Жан Молітор,}

jean.molitor.photography

фотограф-художник, автор проєкту bau1haus, Берлін, Німеччина jean.molitor@bau1haus.de

\section{Abstract}

Research aim is to find out the features of the project bau1haus, which explores the architectural heritage of the Bauhaus in a global context through the art of photography; systematize approaches to the interaction of the photographer with the aesthetics of the building; to record the results of the study in terms of identifying the values of the interaction of the Bauhaus architectural style with the basic principles of architecture of different countries, and its metamorphosis in the present. Research methodology is based on the method of de-
Анотація

Мета статті - з'ясувати особливості проєкту bau1haus, який досліджує архітектурну спадщину Баугаузу у світовому контексті за допомогою мистецтва фотографії; систематизувати підходи до взаємодії фотографа з естетикою будівлі; зафіксувати результати дослідження в аспекті виявлення значень взаємодії архітектурного стилю Баугаузу із засадничими принципами архітектури різних країн, його метаморфози в сучасності. Методологічні засади дослідження базуються на методі деконструкції, що передба- 
construction, which involves the study of visual language in altered conditions with the help of conceptual photography. Scientific novelty. The article considers the place of the Bauhaus in the aspect of the international architectural phenomenon, the style of which is uniquely incorporated into the urban landscapes of different countries and still creates their modern face. The scientific novelty lies in a generalized review of the point events and phenomena during the twentieth century that makes up the evolution of the Bauhaus. The article also presents a unique photographic material - Bauhaus buildings, which were found during the cultural explorations of the photographer J. Molitor in countries of different continents. Some buildings are already in disrepair, others have stopped social time, and others - are restored and functioning in full force. It is important for the photographer to accurately reproduce the image of the building in order to reveal as much as possible its entire socio-cultural context. The study revealed the peculiarities of the photographer's work: his main task is not only to capture a building. It is important to rethink it in preparation for photography and during it in the aspect of modern life, from the perspective of the coexistence of Bauhaus architecture and the man of the globalized digital society. Conclusions. As a result of scientific and artistic research in the framework of the bauthaus project, a network of architects was identified, which becomes clear through the use of similar methods and approaches, as well as influence the general trends of the time. An important result of the bauthaus project is the identification of the interaction of the former aesthetics of the building with modernity, its visual significance in the changed conditions. This was made possible by conceptual photography, which was performed using a systematic approach of the photographer through the understanding of the built-in structure in space. The work done has revealed the essential aspects of modernism, much of which is between the two world wars. The formation, development, and metamorphosis of the forms and methods generated by Bauhaus are also recorded with the help of photography. This is important for the transmission of cultural heritage to future generations and to stimulate further research. чає дослідження візуальної мови у змінених умовах за допомогою концептуальної фотографії. Наукова новизна. В статті розглянуто місце Баугаузу в аспекті міжнародного архітектурного явища, стиль якого унікально інкорпорується в міські ландшафти різних країн і досі створює їхнє сучасне обличчя. Наукова новизна полягає в узагальненому огляді точкових подій та явищ впродовж XX ст., що складають еволюцію Баугаузу. В статті також представлений унікальний фотографічний матеріал - споруди Баугаузу, які були знайдені під час культурологічних розвідок фотографа Ж. Молітора в країнах різних континентів. Деякі будівлі вже знаходяться в аварійному стані, в інших зупинився соціальний час, треті - відреставровані та функціонують у повну силу. Фотографу важливо достовірно відтворити образ будівлі, щоб максимально розкрити весь ії соціально-культурний контекст. У дослідженні виявлено особливості роботи фотографа: його головне завдання не лише фіксувати ту чи іншу будівлю. Важливо переосмислити їі під час підготовки до фотозйомки та під час неї в аспекті сучасного життя, у ракурсі співіснування архітектури Баугаузу та людини глобалізованого цифрового суспільства.

Висновки. У результаті науково-мистецького дослідження у рамках проєкту bauthaus виявлено мережу архітекторів, які стають зрозумілими через застосування подібних методів і підходів, а також впливають на загальні тенденції часу. Важливим результатом проєкту bauthaus є виявлення взаємодії колишньої естетики будівлі із сучасністю, іiі візуального значення у змінених умовах. Це стало можливим за допомогою концептуальної фотографії, що виконана із застосуванням системного підходу фотографа через розуміння вбудованості споруди у простір. Здійснена робота дозволила виявити сутнісні аспекти модернізму, значна частина якого знаходиться між двома світовими війнами. Також за допомогою фотографії зафіксовано становлення, розвиток і метаморфозу породжених Баугаузом форм і методів. Це має значення для передачі культурного спадку майбутнім поколінням та стимулювання подальшої дослідницької роботи. 


\section{Key words: Ключові слова:}

Bauhaus, architecture, conceptual photography, bau1haus.

Баугауз, архітектура, концептуальна фотографія, bau1haus.

\section{Einführung 1}

Einhundert Jahre nach seiner Gründung in Weimar ist «Bauhaus» noch immer das Schlagwort für eine bestimmte Art zu bauen, zu der sich Architekten weltweit bekennen. Ganze Generationen sind mit der sachlich-nüchternen und funktionalen Ästhetik der Moderne konfrontiert und sozialisiert worden. Bauhaus bezeichnet eine Kunstschule, deren großes Ziel eine neue Architektur, hervorgegangen aus einer neuartigen Architektenausbildung war. Das Bauhaus ist nicht der Erfinder der Moderne, aber ein sehr wichtiger Teil davon. Architekt Walter Gropius schrieb anlässlich der Gründung im Jahr 1919: «Das Endziel aller bildnerischen Tätigkeit ist der Bau! Ihn zu schmücken, war einst die vornehmste Aufgabe der bildenden Künste, sie waren unablösliche Bestandteile der großen Baukunst. Heute stehen sie in selbstgenügsamer Eigenheit, aus der sie erst wieder erlöst werden können durch bewusstes Mitund Ineinanderwirken aller Werkleute untereinander. (...) Architekten, Bildhauer, Maler, wir alle müssen zum Handwerk zurück!» (Gropius, 1919). Obwohl Walter Gropius selbst gern das Bauhaus als «Stunde null» beschwor, sind auch seine Ideen zum modernen Bauen Ergebnis eines Prozesses. Es gibt zahlreiche Wegbereiter und Wegbegleiter: Architekten, Künstler, Kunstschulen, Ingenieure, Firmen - national und international. Paris, Wien, Zürich, London, Zagreb, Prag, Moskau, Istanbul oder auch Berlin gehören zum großen Netzwerk der Moderne. Ebenso New York, Tokio und mit der Bauhausaustellung 1923 auch Kalkutta. In den Niederlanden ist es De Stijl, in Prag die Architekten des tschechischen Kubismus, in Turin die italienischen Futuristen, in Mailand die Rationalisten. Selbstverständlich ist es wichtig, die bedeutenden identischen Impulse zu erwähnen, die sich auf dem Gebiet der Sowjetunion entwickelt haben. Es geht nicht nur um die Werkstatten der WChUTEMAS (3 1927 p. WChUTEIN) in Moskau, sondern auch um andere weniger untersuchte, aber nicht weniger wichtige Zentren der Avantgarde, die in der Ukraine (in Kyiv, Charkiw, Odesa) und in Weißrussland (in Wizebsk) von 1920 bis 1930 existierten. Man hat hier im Geiste des kühnen Funktionalismus und manchmal des utopischen Konstruktivismus gearbeitet, bis Stalin einen starren Rahmen für den sozialistischen Realismus schuf.

Die erste Phase des Bauhauses als Zentrum für innovatives Design und Stil wurde im Folgenden fortgesetzt - durch die Auswanderung von Lehrkräften und Schülern und durch die 
Eingliederung in lokale kulturelle Traditionen Und Materialien auf der ganzen Welt verbreitet.

Die Feier des 100-jährigen Jubiläums der Schule ist eine großartige Gelegenheit für die empirische Untersuchung des weltweit verstreuten Bauhaus-Architekturerbes, sowie für die Synthese und Analyse des vorhandenen Materials im Hinblick auf interkulturelle Kommunikation und Globalisierung des frühen 21. Jahrhunderts.

\section{Methoden und Quellen der Forschung}

Die Quellenbasis der Forschung ist zuallererst das einzigartige Fotomaterial welches die Bauhausgebäude abbildet, die während der kulturellen Erkundungen des Fotografen und eines der Autoren des Artikels, Jean Molitor, in Ländern verschiedener Kontinente entdeckt wurde. Die Architekturhistorikerin Kaija Voss, zweite Autorin des Artikels, hat umfangreiche Recherchen, über die fotografierten Bauten hinaus, zu den internationalen Entwicklungslinien und den einzelnen Architekten vorgenommen. Die Forschung basiert daneben auf Studien des Architekten W. Gropius (1925) und weiterer Vertreter der Bauhausschule. Die Archivmaterialen, Fotografien und Materialen, die während der Bauhausaktivitäten veröffentlicht wurden, auch der Katalog der ersten Ausstellung 1923, haben eine bedeutende Rolle in der Forschung gespielt.

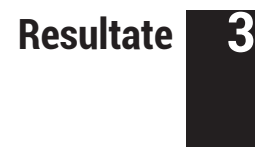

Im neuen Stadium der Bauhaus-Evolution (Ende der 1930er 1940er Jahre), ist das Hauptmerkmal durch die neue Konnotationen des Konzepts und der Ästhetik des Gebäudes in Bezug auf Auswanderung und Exil von Architekten, die die Ideen der Schule unterstützen, gekennzeichnet. Renommierte jüdische Architekten wie Erich Mendelsohn, Erwin Gutkind, Marcel Breuer, Bruno Ahrends oder Fritz Landauer waren gezwungen Deutschland zu verlassen, so wie viele andere kreative, junge und gut ausgebildete Baufachleute. Die Weiße Stadt in Tel Aviv, gegründet 1909 als Siedlung jüdischer Einwanderer vor den Toren von Jaffa, wurde zum Zentrum der Immigration und hatte 1938 bereits 150.000 Einwohner. Viele der angekommenen, größtenteils deutschstämmigen Juden, hatten am Bauhaus studiert oder waren auf andere Weise mit den Ideen der architektonischen Moderne in Kontakt gekommen. Sie gestalteten als Zeichen des politischen Neuanfangs moderne neue Stadtviertel. Mit mehr als 4.000 Bauten entstand die weltweit größte Ansammlung von Häusern im Bauhausstil.

Die internationale Erfolgsgeschichte des Bauhauses, die weltweite Verbreitung seiner Ideen und Protagonisten gipfelte in der Emigration der drei Direktoren, Walter Gropius, Hannes Meyer, Ludwig Mies van der Rohe und zahlreicher Bauhausmeister und Studenten. Sie trugen die Ideen der Schule um die Welt. Ebenso weitere Architekten, die der Moderne eng verbunden waren, wie 
Ernst May, Bruno Taut oder Martin Elsaesser, suchten ihre Aufträge während der nationalsozialistischen Diktatur im Ausland. In den 1950er Jahren kehrten die Bauhausideen als Nachkriegsmoderne zurück nach Deutschland. Unter anderem Hans Scharoun, Arnold Blauvelt, Hans Schwippert oder Otto Bartning waren es, die sich um ein modernes Gesicht Deutschlands verdient machten. Bauausstellungen wie die Berliner Interbau 1956/57 wollten das moderne offene Deutschland zeigen. International arbeitende Architekten wie Walter Gropius, Ludwig Mies van der Rohe oder Le Corbusier halfen beim Neuaufbau in Europa, ebenso Oskar Niemeyer oder Alvar Aalto und deutsche Architekten wie Sep Ruf, Max Taut, Gustav Hassenpflug, Robert Vorhoelzer oder Egon Eiermann.

Weiße Bauten mit Fensterbändern und flachen Dächern und die von der Industrie geprägte Ästhetik sind zur Alltäglichkeit geworden. Fragen nach dem sozialen Wohnungsbau stellen sich bis heute, vor allem in den großen Ballungsräumen von Deutschland in Städten wie München, Frankfurt am Main, Stuttgart, Berlin oder Hamburg. Damit einher ging auch ein Verlust der in den 1920er Jahren angestrebten Perfektion. Die zunächst konsequente Hinwendung zu kühnen Formen aus Schiffsbau, Autoindustrie oder Maschinenbau war nicht immer umzusetzen. Die Ideen der Architekten wurden von Notwendigkeiten der Baugesetzgebung und Normierung, von Finanzierungsfragen sowie der Kritik am zu «kalten» und zu «nüchternen» Bauhaus hinweggespült. Bauhaus-Ästhetik kann auch Disziplin erfordern. Bauherren und Nutzer sind nicht immer dazu bereit, der Entwurf erfährt Veränderungen, die Moderne Kritik.

Nach dem 2. Weltkrieg wurde 1953 in Ulm die Hochschule für Gestaltung (HfG Ulm) von Inge Aicher-Scholl, Otl Aicher und Max Bill gegründet. Walter Gropius hielt die Eröffnungsrede, ein Fortführen des praktischen Bauhaus-Modells der Werkstätten war zunächst geplant. Es gab keine Architekten - sondern eine Designerausbildung. Doch setzte sich ein theoretisch-wissenschaftliches Studienmodell durch. Die Hochschule für Gestaltung Ulm bestand bis 1968 und gilt als die international bedeutendste Design-Hochschule nach dem Bauhaus. In Weimar entstand ab 1954 die Hochschule für Architektur und Bauwesen Weimar, heutige «Bauhaus-Universität Weimar», unter anderem Ausbildungsstätte für Architektur und Mediendesign.

1960 gründete Hans Maria Wingler in Darmstadt das Bauhaus Archiv. Unterstützung erhielt er dabei durch Gropius. 1979 zog das Archiv nach Berlin. Das Gebäude war 1964-68 von Walter Gropius, Wils Ebert, Alexander Cvijanovic und Louis McMillen für einen Standort in Darmstadt mit Hanglage konzipiert worden. Es wurde an die topographischen Verhältnisse von Berlin, den flachen Standort am Landwehrkanal, angepasst. Gropius verstarb 1969, die formalen Merkmale seines Baus, wie Rampe und Sheddächer wurden 
beibehalten - eine Würdigung seines signifikanten Entwurfes. Seit 1986 wurden die Bauhaus-Gebäude in Dessau vom «Bauhaus Dessau - Zentrum für Gestaltung» genutzt, heute ist es Sitz der 1994 gegründeten Stiftung Bauhaus Dessau im Land SachsenAnhalt.

Der Zeitgeist der Moderne hatte sich spätestens in den 1940er Jahren auf der ganzen Welt verbreitet, adaptiert an die klimatischen Verhältnisse, an Bautechniken und Baumaterialien vor Ort. Nahezu überall gibt es, manchmal sehr überraschende Spuren der Moderne: in Argentinien, Burundi, Chile, Guatemala, Indien, Indonesien, Israel, Kambodscha, Kenia, Kuba, Rumänien, Russland. Das Bauhaus als Prozess der Moderne, verträgt sich scheinbar mühelos mit dem heutigen Zeitgeist und der Architektur weltweit. Architekten wie Frank O. Gehry, Daniel Libeskind oder das Büro COOP Himmelb(I)au, die dynamische, sich scheinbar bewegende Strukturen bauen, dekonstruieren die allgegenwärtigen Formen der Moderne. Architekten wie Richard Meier, Norman Foster, Murphy Jahn Architects, Stefan Braunfels, das japanische Büro SANAA oder die Schweizer Herzog \& de Meuron sind wie ganze Architektengenerationen mit dem Bauhaus und dem industriellen Bauen sozialisiert worden. Bis heute entstehen täglich Gebäude in den Formen der Moderne. Qualitativ hochwertige Architektur der Moderne, von ihren Anfängen zu Beginn des 20 Jahrhunderts bis heute aufzuspüren und im Bild festzuhalten, ist Aufgabe des Projektes bau1haus. «Mein Ziel ist ein weltweites Fotoarchiv dieser Gebäude», sagt der Berliner Fotograf Jean Molitor. Verbindungen zwischen den einzelnen Strömungen einer von vielen Impulsen gekennzeichneten Zeit und eines bislang kaum dargestellten Netzwerkes kreativer Architekten sollen aufgezeigt werden. Die «Vergleichende Ästhetik der Moderne» - eine fotografische Gegenüberstellung von Bauten aus unterschiedlichsten Teilen der Welt, über Kontinente hinweg, steht an erster Stelle des Projektes bauThaus.

\section{Das Projekt bau 1 haus in der Vision von Jean Molitor.}

In den zurückliegenden Jahren der intensiven Recherche und der Arbeit vor Ort konnte bauThaus seinen Platz im Bereich der hochwertigen Architekturfotografie finden und gut seinen Ansatz der vergleichenden Ästhetik vertreten, welcher in den ersten Jahren allgemein eher skeptisch in der Fachwelt gesehen wurde. In diesem fotografischen Projekt ist es nicht die Hauptsache, sich auf die Form, architektonische Details oder strukturelle Merkmale zu konzentrieren. Wenn das Format zunächst Interesse weckte, dann im Zuge der vorbereitenden Analytik. Bis heute hat sich jedoch viel getan, der oberflächlich vergleichende Ansatz ist zu einer wissenschaftlich anerkannten Formstudie gewachsen. Die Gebäude stehen nicht losgelöst, sondern sind textlich eingebettet in den einstigen Zeitgeist und die sich verändernden Möglichkeiten von 
neuen Werkstoffen und Technologien. Auch wenn sich das Projekt dem Bauhaus selbst sehr verbunden fühlt, offenbart es in seinem globalen Ansatz eine bislang eher im Verborgenen gebliebene Formenvielfalt, eine Art Architektur-Familie. Das Zusammenwirken unterschiedlichster avantgardistischer Strömungen, ausgehend von Europa und den USA wird sichtbar. Die Definition «das Patchwork der klassischen Moderne» kann auf ein solches Phänomen angewendet werden. Einige Gebäude, welche die Ideen und den Stil des Bauhauses tragen, sind echte Entdeckungen, auf Reisen oder im Archiv. Zum Beispiel das Gebäude der Kirche Be Maro in Guadeloupe, aufgespürt während einer Forschungsreise nach Bass Terre im Jahr 2020. Das Gebäude erbaute der französische Architekt Ali Georges Tur im Jahre 1936, der einst von der Regierung in Paris auf die kleinen Antillen gesandt wurde. Er hat dort nach der verheerenden Naturkatastrophe im Stile des europäischen Zeitgeistes mit karibischem Einfluss vor allem betroffene öffentliche Gebäude wieder auf- bzw. neugebaut. So kam es besonders in Guadeloupe zwischen 1929 und 1937 zu einer fast radikalen Veränderung des Baustils, welcher noch heute das Stadtbild prägt.

2019, anläßlich des hundertjährigen Bestehens des Bauhaus und dem sich daraus ergebenden öffentlichen Interesse wurden Teile der Sammlung in 24 Ausstellungen gezeigt, darunter an Orten wie Havanna, Vancouver oder im Zusammenhang mit einem Goethe-Stipendium in Ankara.

Da die Fotografie eine wichtige Rolle im Projekt spielt, war es notwendig, bestimmte Ansätze für die Arbeit des Fotografen zu entwickeln, der eine einzigartige Basis architektonischer Aspekte zusammenführt und die Präsenz dieses Erbes in der Neuzeit überdenkt. Zunächst soll ein systematischer Ansatz verfolgt werden, um die gebaute Struktur als einen Raum zu verstehen, in dem Kommunikation, natürliche Elemente, alle Zeichen des menschlichen Lebens zusammengeführt sind. Ein gutes Beispiel dafür ist das Wohnhaus der Ärztekooperative nahe der MetroStation Soloti Worota in Kyiv. Das Gebäude wurde zwischen 1928 und 1930 und vom Architekten Pawel Aleshin erbaut. Die Situation von bebautem Umfeld und Verkehrssituation hat sich seit den 1930er Jahren stark gewandelt.

Die interessantesten Bilddokumente entstehen in der Regel kurz nach der Erbauung, da sie einen freien Blick auf den Baukörper gestatten und Faktoren wie Elektrifizierung mit Kabelleitungen und Masten, parkende Autos, üppige Vegetation, umgebaute Balkone, Abfallcontainer oder unentwegter Passantenverkehr den Blick des Betrachters nicht ablenken.

Die folgenden Ausgangsbedingungen sind für die Durchführung eines solchen Projekts sehr wichtig: 
1. Sorgfältige Vorbereitung und Vorhersage der fotografischen Bedingungen

Das Fotografieren heute muss oft in einem begrenzten Zeitrahmen stattfinden, und wieder wichtig, das Wetter muss stimmen da Anreise und Aufenthalt aufwendig und kostenintensiv sind. So geschehen 2018 bei der Reisenach Israel: EineknappeWoche für die Städte Rehovot, Jerusalem, Haifa und Tel Aviv. Ein gewaltiges Pensum, begleitet von Dauerregen. Entstanden sind lediglich Vorarbeiten zu einer detaillierten Recherche vor Ort, in welcher der genaue Sonnenstand, die konkrete Anfahrt im Land, die GPS-Daten zur Lokalisierung, mögliche Genehmigungseinschränkungen und im besten Fall ein Ansprechpartner in einem Datenblatt vermerkt sind.

2. Umweltintegrierter Ansatz

Oft wird in dem Zusammenhang bei Vorträgen oder Diskussionsrunden gefragt, ob mit einer generellen Aufnahme das Ziel nicht bereits erreicht sei oder es stellt sich die berechtigte Frage, ob Architektur nicht von Menschen für Menschen gebaut ist und so das belebende Moment unserer eigenen Zeitebene durchaus berechtigt sei. Dem kann man grundsätzlich zustimmen, wenn es sich um ein dokumentarisches Projekt handelt. Die Grundlage bildet das Genre der traditionellen Street Photography. In diesem Bereich ist es wichtig, das fotografierte Abbild inhaltlich zu gestalten im Sinne von Brennweite, Schärfe-Unschärfe oder der eigenen Interpretation vom goldenen Schnitt - aber das Leben, die Situation, die Landschaft in ihrer Wirklichkeit einzufangen, als Abbild ihrer Zeit, als möglichst objektiver Beleg für die Nachwelt. In bauThaus liegt der selbstgewählte Schwerpunkt anders. Hier handelt es sich um konzeptionelle Fotografie mit künstlerischer Ausrichtung und nachgeordnetem dokumentarischen Anteil. Die Architektur steht im Vordergrund, sie geradezu zeitlos zu präsentieren ist das Ziel. Von Ablenkungen frei, soll sie den Blick auf das Wesentliche leiten, die Idee der Linie, das Spiel der Baukörper als Teil einer gesamten architektonischen Komposition im Sinne ihrer Zeit. In diesem Arbeitsgedanken wird auf Neuinszenierungen durch extremes Licht, wo Schatten und Sonnenpartien das Objekt mitunter bravourös in Szene setzen, jedoch die Vergleichbarkeit als wesentlichen Punkt dieser Arbeit erschweren, verzichtet.

3. Durchführung von Recherchen zum Thema und die reale Situation

Im Vorfeld der Fotografie muss unbedingt ein umfangreicheS Archiv- und Literaturstudium erfolgen. Neben den Recherchen und Studien der Architekturhistorikerin Kaija Voss, wird die zweite Ebene durch ein in 10 Jahren gewachsenes Netzwerk an freiwilligen Mitarbeitern, von Architekten in der ganzen Welt, Universitäten und Instituten oder auch Freunden des Projektes gebildet. Die dritte und 
wichtigste Ebene bleibt das Schauen vor Ort. Sich selbst ein Bild zu machen, dem über Jahre geschulten Blick zu vertrauen und auf Entdeckungsreise zu gehen, den direkten Dialog mit den Menschen, Bewohnern und Behörden nicht zu scheuen, bleibt für bauThaus unersetzlich und entspricht somit auch wieder dem Gedanken der Street Photography.

In Bezug auf die Arbeit im Projekt, so ein Autor des Artikels, Jean Molitor, geht es nicht um das formale Abarbeiten einer imaginären Bildliste, die allerdings gefühlt bereits unendlich ist. Das Reisen zu den Menschen und Orten wird nach wie vor als ein Geschenk angesehen, als etwas Wertvolles für uns in jeder Zeit. Das Ziel ist anzukommen, gelebte Architektur im Wandel der Zeit zu erfahren und im friedvollen Miteinander austauschend zu kommunizieren. Aus diesem wertvollen Gedanken und der gemachten Erfahrung speist sich bauthaus. Und genau das soll sich auch nicht ändern. Wenn der Autor des Artikels bislang «nur» für dieses Projekt in 40 Ländern fotografisch tätig war, ist dies im Nachhinein kaum vorstellbar. Was bedeutet es für die Zukunft, wenn bislang Gebäude der sogenannten Klassischen Moderne in ihrer unterschiedlichsten Ausprägung in 108 Staaten recherchiert sind und das Recherchierte selbst nur ein geringer Teil des Vorhandenen ist? So finden sich in der Forschung auch global Gebäude in neuer sozialer Ausrichtung, technologischem Fortschritt oder mit Freizeittendenzen nach dem ersten Weltkrieg wie die Feuerwehrstation in Miami, eine Schule in Berlin, ein Planetarium in Istanbul, eine Fabrik in Dresden, eine Villa in Gitega, ein Theater in Battambong, eine Tankstelle in Asmara, eine Radiostation in Guatemala-Stadt, ein Arbeiterklub in Moskau, ein Krankenhaus in Tel Aviv, eine Fleischfabrik in Buenos Aires, eine Synagoge in Havanna. Die Liste ist schon heute mehrere Meter lang und es wird Zeit für eine Datenbank um einen konzentrierten Zugriff zu haben, um eines Tages das Projekt der Öffentlichkeit und den Studierenden zur Verfügung zu stellen. Die Erforschung der Hintergrunddaten wie Architekten, Baujahre oder Verwendungszweck steht parallel im Fokus und verbindet die künstlerische Arbeit mit wissenschaftlichen Komponenten. Ein geradezu unglaubliches Netzwerk von Architekten offenbart sich, Verbindungslinien wie auch Beeinflussungen bis hin zu allgemeinen Trends jener Zeit werden deutlich. Ziel wird es sein, die Moderne mit ihrem Hauptteil zwischen den beiden Weltkriegen in seiner globalen Ausdehnung zu erfassen und als veranschaulichte Studie kommenden Generationen zu überliefern und zu aufbauenden Arbeiten anzuregen. Zudem strebt bauThaus auch in enger Zusammenarbeit mit docomomo den internationalen Diskurs über die Moderne als Welterbe an. bau Thaus präsentiert sich derzeit rund um den Globus mit Wanderausstellungen. Über Webcams kann die Vernetzung der weltweiten Standorte und somit eine unserem 
Zeitgeist entsprechende Präsentationen erreicht werden. Begleitet werden die Ausstellungen durch Vorträge und Bücher, Presseartikel und Filme. Interviews mit Zeitzeugen und Diskussionen zur aktuellen Baukultur sind in konsequenter Vorbereitung.

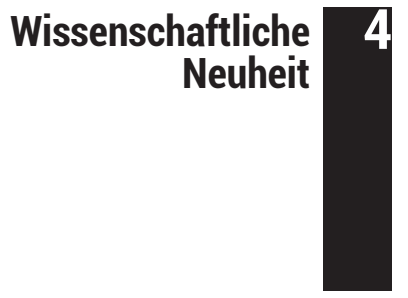

Der Artikel betrachtet den Platz des Bauhauses im Hinblick auf das internationale Architekturgeschehen, als Institution, dessen Bauten in einzigartiger Weise in die Stadtlandschaften verschiedener Länder integriert sind und darüber hinaus ihr modernes Gesicht prägen. Die wissenschaftliche Neuheit liegt in einer allgemeinen Übersicht über Punktereignisse und -phänomene im 20. Jahrhundert, welche die Entwicklung des Bauhauses ausmachen. Der Artikel präsentiert auch ein einzigartiges Fotomaterial, die Gebäude, die während der kulturellen Erkundungen des Fotografen Jean Molitor in Ländern verschiedener Kontinente gefunden wurden. Einige Gebäude sind bereits in einem schlechten Zustand, in anderen hat die «soziale Zeit» gestoppt, noch andere Gebäude sind restauriert und funktionsfähig. Für den Fotografen ist es wichtig, das Bild des Gebäudes genau wiederzugeben, um den gesamten soziokulturellen Kontext so gut wie möglich zu offenbaren. Die Forschung zeigt die Besonderheiten des Fotografen auf, dessen Hauptaufgabe es ist, ein Gebäude nicht nur einzufangen, sondern es auch in Vorbereitung auf die Fotografie und während des modernen Lebens im Hinblick auf das Zusammenleben von Bauhaus-Architektur und globalisierter digitaler Gesellschaft zu überdenken.

\section{Schlussfolgerung 5}

Als Ergebnis wissenschaftlicher und künstlerischer Forschung im Rahmen des Projekts «Das Projekt bau1haus» wurde ein Netzwerk von Architekten identifiziert, das im Kontext der Verbindung mit dem Bauhaus und den damaligen Trends deutlich wurde. Die Arbeit ermöglichte es, das globale Ausmaß der Moderne mit ihrem Hauptteil zwischen den beiden Weltkriegen zu verstehen und es als Studie an zukünftige Generationen weiterzugeben und weitere Arbeiten anzuregen. Ein wichtiges Ergebnis von bauThaus ist die Identifizierung des Zusammenspiels der früheren Ästhetik des Gebäudes mit der Moderne, ihrer visuellen Bedeutung unter den veränderten Bedingungen. Möglich wurde dies durch die zahllosen brillianten Fotos, die mit dem systematischen Ansatz des Fotografen weltweit aufgenommen wurden. 
Деміург: ідеї, технології, перспективи дизайну 2020 Том 3 № 1 Demiurge: ideas, technologies, perspectives of design 2020 Vol. 3 No 1
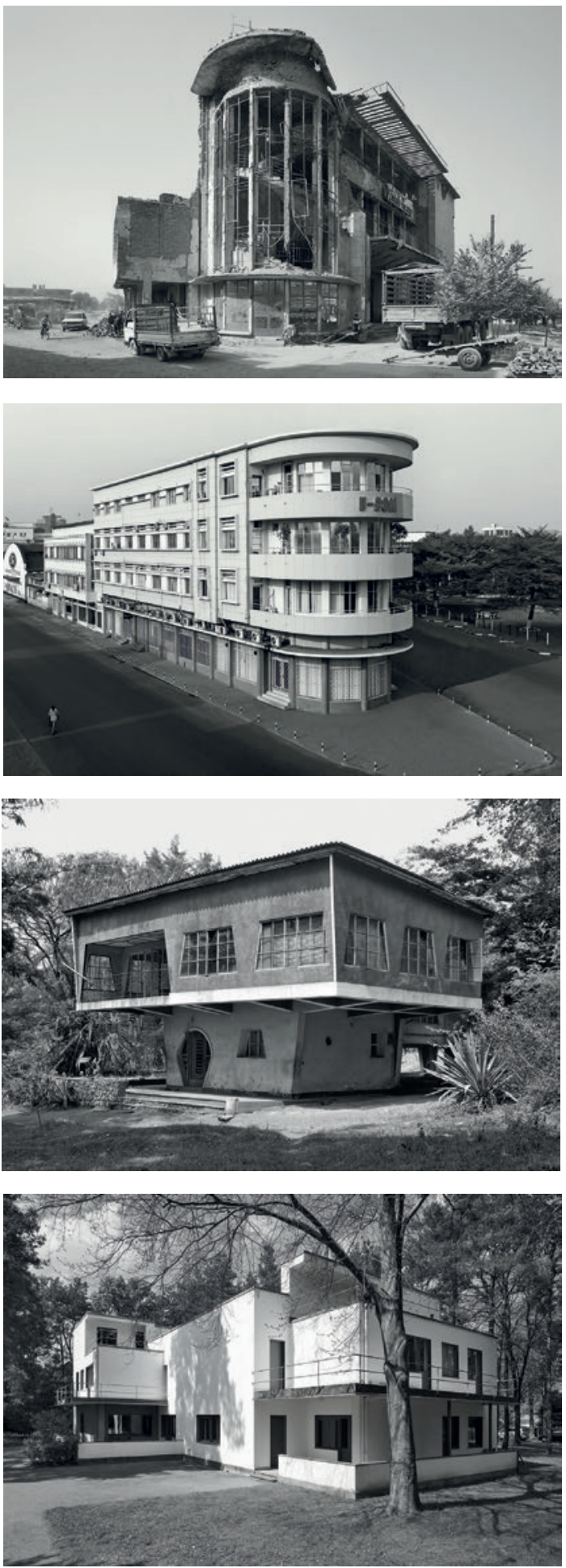

Bild 1. Unbekannt Architekt. Kino. Barikot. Kabul. Afghanistan. Um 1930.

Foto: J. Molitor, 2007.

Рис. 1. Невідомий архітектор.

Кінотеатр. Барикот. Кабул. Афганістан. Близько 1930 р. Світлина Ж. Молітора, $2007 p$.

Bild 2. Unbekannt Architekt. Telefongesellschaft U-com. Bujumbura. Republik Burundi. Um 1940-46. Foto: J. Molitor, 2009.

Рис. 2. Невідомий архітектор Телефонна компанія U-com. Бужумбура. Республіка Бурунді. Близько 1940-46 рр.

Світлина Ж. Молітора, 2009 р.

Bild 3. Unbekannt Architekt. Wohnhaus Le Champignon. Gitega. Republik Burundi. Um 1940-46. Foto: J. Molitor, 2009.

Рис. 3. Невідомий архітектор. Будинок Le Champignon. Гітега. Республіка Бурунді. Близько 1940-46 рр. Світлина Ж. Молітора, 2009 р.

Bild 4. Walter Gropius. Meisterhaus. Dessau. Deutschland. 1926.

Foto: J. Molitor, 2014.

Рис. 4. Вальтер Гропіус. Будинок майстра. Дессау. Німеччина. 1926. Світлина Ж. Молітора, 2014 p. 

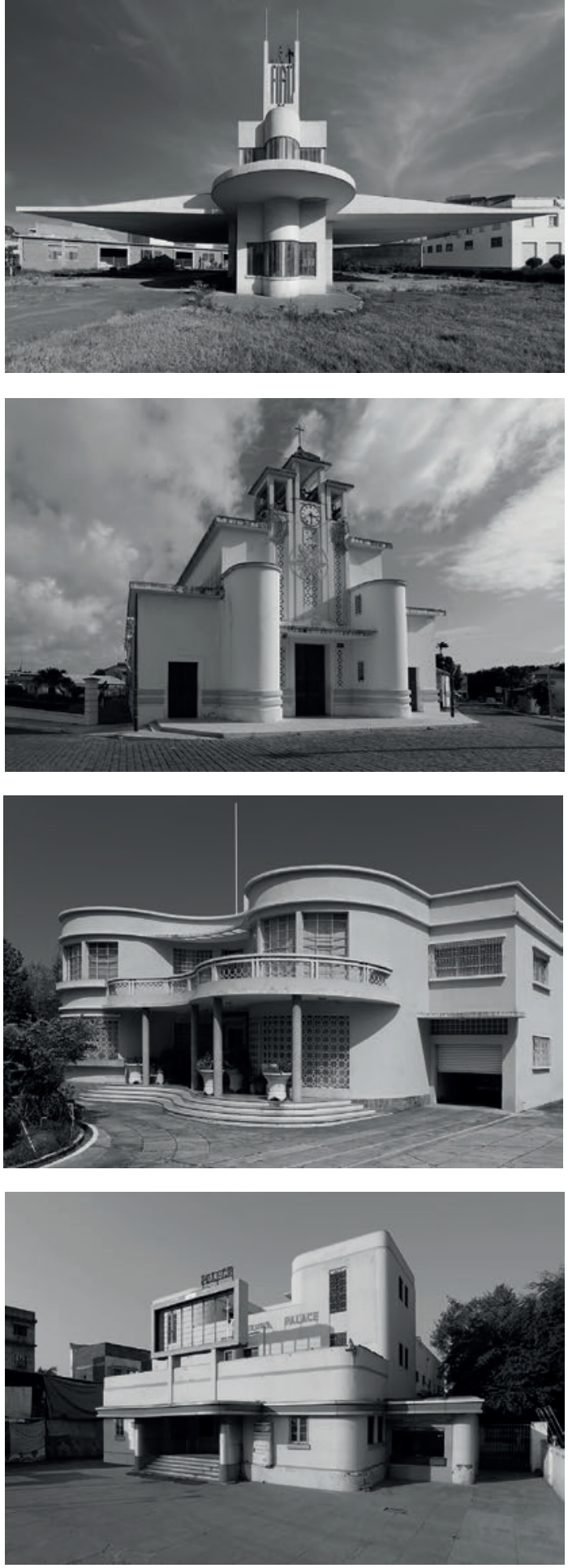

Bild 5. Guiseppe Pettazzi. Tankstelle Fiat Tagliero. Asmara. Eritrea. 1938. Foto: J. Molitor, 2019.

Рис. 5. Джузеппе Петацці. Заправка Fiat Tagliero. Асмара. Еритрея. 1938. Світлина Ж. Молітора, 2019 р.

Bild 6. Ali Georges Tur. Kathedrale Baie Mahault. Basse Terre. Guadeloupe.

Kathedrale. 1936. Foto: J. Molitor, 2020.

Рис. 6. Алі Жорж Тур. Собор Ваіе Mahault. Бас-Тер. Гваделупа. 1936. Світлина Ж. Молітора, 2020 р.

Bild 7. Unbekannt Architekt. Wohnhaus. Guatemala-Stadt. Guatemala.

Um 1940-50. Foto: J. Molitor, 2017.

Рис. 7. Невідомий архітектор.

Житловий будинок. Гватемала-Сіті. Гватемала. Близько 1940-1950 pp. Світлина Ж. Молітора, 2017 р.

Bild 8. Unbekannt Architekt. Kino Picture Palace. Udaipur. Indien. Um 1940.

Foto: J. Molitor, 2017

Рис. 8. Невідомий архітектор. Кінопалац. Удайпур. Індія. Близько 1940 р. Світлина Ж. Молітора, 2017 р. 
Деміург: ідеї, технології, перспективи дизайну 2020 Том 3 № 1 Demiurge: ideas, technologies, perspectives of design 2020 Vol. 3 No 1
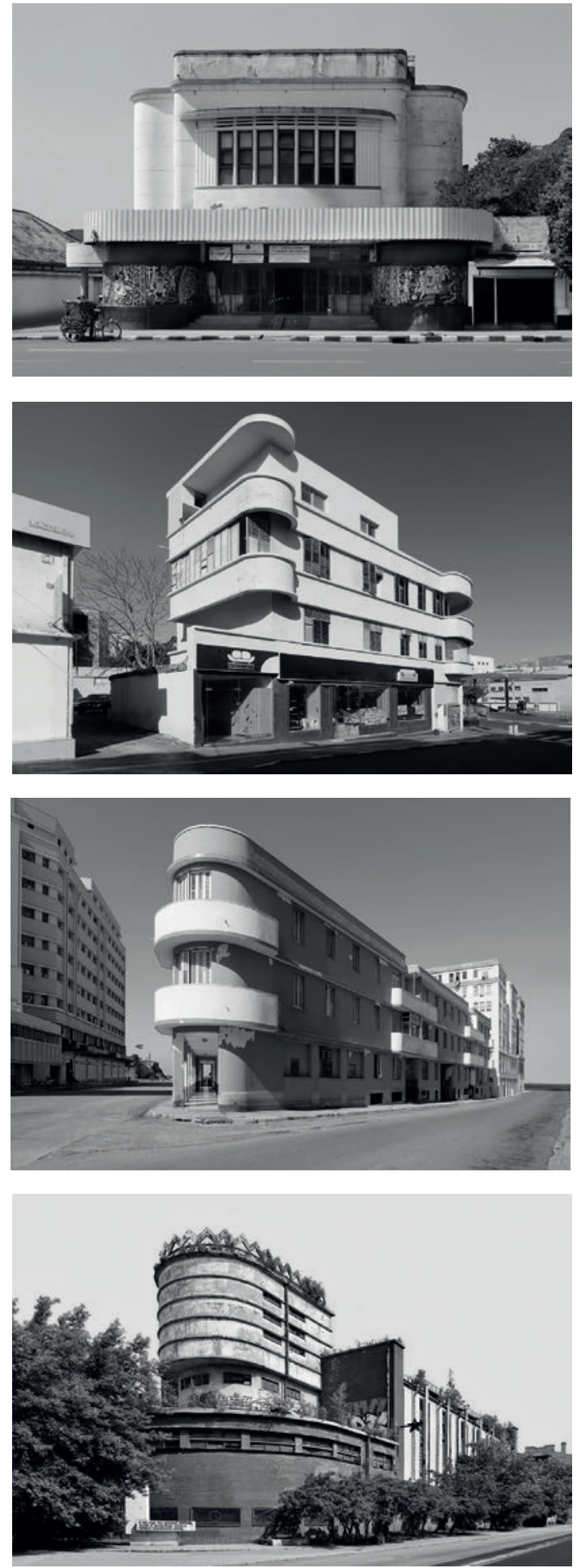

Bild 9. Unbekannt Architekt. Kino Dian. Bandung. Indonesien. Um 1935.

Foto: J. Molitor, 2015

Рис. 9. Невідомий архітектор. Кінотеатр Dian. Бандунг. Індонезія. Близько 1935 р. Світлина Ж. Молітора, $2015 p$.

Bild 10. Unbekannt Architekt. Wohnhaus. Haifa. Israel. Um 1935. Foto: J. Molitor, 2018.

Рис. 10. Невідомий архітектор. Житловий будинок. Хайфа. Ізраїль. Близько 1935 р. Світлина Ж. Молітора, $2018 p$.

Bild 11. Unbekannt Architekt. Residencia Canteras. Havanna. Kuba.

Um 1936. Foto: J. Molitor, 2019.

Рис. 11. Невідомий архітектор.

Резиденція Canteras. Гавана. Куба. Близько 1936. Світлина Ж. Молітора, $2019 p$.

Bild 12. Erich Mendelsohn. Textilfabrik «Rotes Banner». St. Petersburg. Russland. 1925-26. Foto: J. Molitor, 2014.

Рис. 12. Еріх Мендельсон. Текстильна фабрика «Червоний прапор». Близько 1925-26 рр. Світлина Ж. Молітора, $2014 p$. 


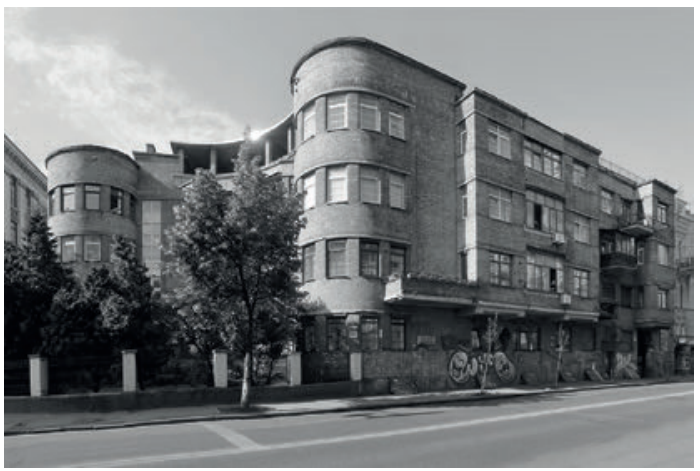

Bild 13. Pawlo Aleshin. Wohnhaus der Aerzte. Kyiv. Ukraine. 1928-30.

Foto: J. Molitor, 2019.

Рис. 13. Павло Альошин.

Будинок лікаря. Київ. Україна.

1928-30 рр. Світлина Ж. Молітора, $2019 p$.

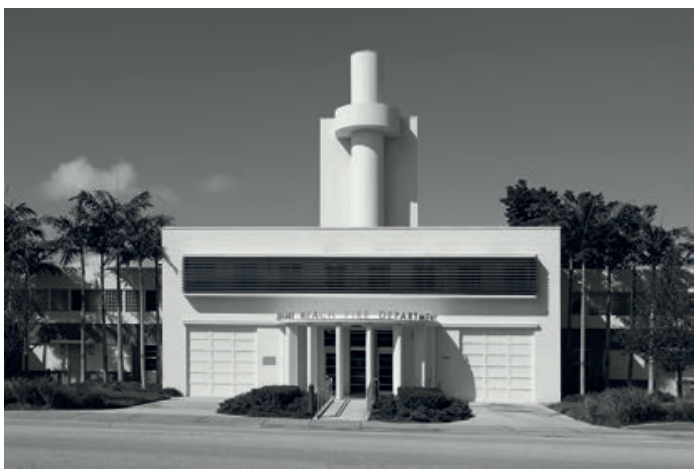

Bild 14. Feuerwache. Miami. USA. 1939.

R. L. Weed, E. T. Reeder.

Foto: J. Molitor, 2013.

Рис. 14. Пожежна станція. Маямі.

США. 1939. Р. Л. Віід, Е. Т. Рідер.

Світлина Ж. Молітора, 2013 р.

\section{References}

Gropius, W. (1919). Bauhaus Manifest. Bauhausbookshelf. https://www.bauhaus-bookshelf.org/ [in German]

Gropius, W. (1925). Internationale Architektur [International architecture] (Bauhausbuch 01). Albert Langen Verlag [in German].

Jean Molitor - bau1haus: project and photographs. (2019). Bauhaus \& modernism. https://www. bauhausmoderne.org/ [in English].

Molitor, J. (2019). Bauhaus is a household name in the general public. Interview Bauhaus 100. https:// www.bauhaus100.com/magazine/understand-the-bauhaus/bauhaus-is-a-householdname-in-the-general-public/ [in English].

Molitor, J., \& Voss, K. (2018). Bauhaus. Eine fotografische Weltreise. A photographic journey around the world [Bauhaus. A photographic trip around the world. A photographic journey around the world]. Be.bra verlag [in German].

Staatliches Bauhaus in Weimar 1919-1923. Katalogbuch [Staatliches Bauhaus in Weimar 19191923. Catalog book]. (1923). Albert Langen Verlag [in German]. 